\title{
Pitfalls for diagnosis of burning mouth-like syndrome
}

\author{
Raluca Ema Pirvu', Ioanina Parlatescu' ${ }^{1}$, Serban Tovaru', Cosmin Dugan², Paula Perlea ${ }^{3}$ \\ ${ }^{1}$ Department of Oral Pathology, Faculty of Dental Medicine, \\ "Carol Davila" University of Medicine and Pharmacy, Bucharest, Romania \\ ${ }^{2}$ Department of Neurology, Faculty of Medicine, "Carol Davila" University of Medicine and Pharmacy, Bucharest, Romania \\ ${ }^{3}$ Department of Endodontics, Faculty of Dental Medicine, "Carol Davila" University of Medicine and Pharmacy, \\ Bucharest, Romania
}

\begin{abstract}
This research gives a scientific framework for burning mouth syndrome(BMS) etiology and diagnostic approach in clinical dental and medical practice. BMS-like symptoms can be induced by systemic diseases such as diabetes, gastrointestinal, endocrine disorders, allergy etc or by local oral cavity conditions as candidiasis or geographic tongue or odontogenic causes. Because the etiology of BMS is multifactorial, treatment can only be distinctive, and is aimed at relieving symptoms. The complexity of BMS symptoms and associated psychosocial infirmities, anxiety and depression raise the need for a multidisciplinary and individualised approach.
\end{abstract}

Keywords: burning mouth syndrome, oral mucosa, burning mucosa, oral dysesthesia

\section{INTRODUCTION}

The World Health Organization has defined burning mouth syndrome (BMS) as a "chronic orofacial pain with an intraoral burning or dysaesthetic sensation that recurs for more than 2 hours per day on $50 \%$ of the days over more than 3 months, without evident causative lesions on clinical investigation and examination" [1]. It has a multifactorial etiology and is associated with psychological distress such as anxiety, depression, rage/ frustration. The diagnosis is established by exclusion of other diseases that would better explain the symptoms [1].

In the literature there are four types of classification for BMS. For a uniform reporting,the oral medicine specialists and dental practitioners use mainly the first one, which is based on the evolution of BMS symptoms. The second classification refers also to the clinical criteria, mainly the associated symptoms. The third classification divides BMS in subclinical neuropathies and the fourth is based on etiology.
Based on symptoms evolution BMS is classified in three types [2,3]. In BMS type 1 the symptoms are not present on waking up, but appear and increase in severity as the day progresses, without preventing the patient from falling asleep; according to the authors, this type is observed in approximately $35 \%$ of BMS patients. In BMS type 2 symptoms are present continuously, all day after waking up; this type is encountered in approximately 55\% BMS patients. Type 2 BMS patients are the most difficult to treat successfully, because a large proportion have chronic anxiety, which is the most recalcitrant obstacle to cure. In BMS type 3, patients have asymptomatic days and may experience symptoms in unusual oral sites, such as the floor of the mouth, oral mucosa, and throat. $10 \%$ of BMS patients have this history, which in the past has been reported as "atypical" heartburn. A study of type 3 BMS patients determined that their main precipitating factors were emotional instability and allergic factors [4].

The second classification is based on the presence of associated oral symptoms and divides BMS 
as follows: (1) complete BMS (oral mucosal pain, dysgeusia, and xerostomia), (2) oligosymptomatic BMS (pain and dysgeusia, or pain and xerostomia), and (3) monosymptomatic pain) [5]. In addition, the authors introduced a distinction in theory symptomatic criteria and additional symptomatic criteria.

The third classification is based on subclinical neuropathies associated with BMS [6]. It divides BMS patients in a subgroup characterized by peripheral neuropathy with small diameter fibers of the oral mucosa and a subgroup that fits the concept of central pain. The two types can overlap in some cases. Individuals with the peripheral type of BMS respond better to local treatments, while the central type does not respond to local treatments and is often associated with psychiatric comorbidity, such as depression, anxiety, and cancerophobia [7]. Lately another subclinical neuropathic subtype was added to the previously mentioned types. This BMS type includes patients with lingual, mandibular or trigeminal subclinical pathology, which will be determined after careful neurophysiological examination and is clinically indistinguishable from previous groups [7].

The etiological classification [8] recognize three subtypes of BMS that support the suspected etiology: BMS type 1 caused by systemic factors, including side effects of medications; BMS type 2 produced by neurological imbalance, during which the symptoms are caused by central or peripheral neurological dysfunctions that affect, in particular, the taste path; BMS type 3 by idiopathic cause.

The International Headache Society (IHS) in the third edition of Classification of Headache Disorders stated that BMS is a matter of debate in patients with general diseases or local factors [9]. In the diagnostic criteria of BMS, IHS included the presence of symptoms for $>2$ hours/day for $>3$ months with burning or dysaesthetic sensation involving the superficial oral mucosa [9].

A variety of local and systemic diseases, including allergies and side effects of medications, can cause burning sensations in the mucosa. Their symptoms are not usually very different from those of BMS. These cases mimic BMS and were previously named secondary BMS or secondary to other conditions by Scala [10]. Secondary BMS cases are different from "primary" or "idiopathic" BMS in which a distinct etiology cannot be determined. The idiopathic BMS is a diagnosis of exclusion of all possible general and local causes for burning sensation. From the first consultation and suspicion of BMS, a complete set of laboratory investigations is recommended. These tests include a complete haematological evaluation (complete blood count, hemoglobin, serum iron, zinc, ferritin, vitamin B1, B2, B6, B12, and folic acid), serum glucose, thyroid panel, antibodies to Helicobacter pylori, etc. In patients with diagnosed systemic disease, a medical consultation for its treatment is recommended.

\section{BURNING MOUTH LIKE-SYNDROME AND SYSTEMIC DISEASES}

\section{Gastrointestinal disorders}

In a large number of BMS patients (37.5\% of 884) were detected the presence of serum gastric parietal cell antibody, thyroglobulin antibody, thyroglobulin antibody positivity. Further studies are needed to determine if gastric parietal cell autoantibodies are detected in BMS patients which can be thus diagnosed with autoimmune atrophic gastritis [11]. Also gastroesophageal reflux disease may be involved in the development of BMS. The use of appropriate treatment given the reflux characteristics was associated with an improvement in symptoms [12]. In BMS patients with gastric disorders, a gastroenterologist evaluation is needed.

There are several reports in the literature in which celiac disease is associated with BMS-like symptoms. Such a case of an elderly woman suffering from a burning tongue may not meet the diagnostic criteria for BMS, it has been reported. Complete relief of symptoms was observed 10 weeks after initiating a gluten-free diet [13].

\begin{abstract}
Allergy
In rare cases of BMS-like symptoms, patients may experience allergies, for example to peppermint oil, chewing gum, toothpaste, mouthwash and lipstick. Although this subtype may be associated with dental allergens, several food, cosmetic and pharmaceutical products have also been identified as allergens related to the occurrence of this "atypical" BMS or BMS type [14].
\end{abstract}

\section{Blood disorders}

A series of blood disorders associate the burning sensation of the oral mucosa and particularly the tongue.

In the general population it has been estimated that a few percent of older men and probably 10$20 \%$ of older women suffer from iron deficiency anemia [10]. Oral findings suggesting the presence of iron deficiency include cracks or fissures of the labial commissures, a pallor colour of the oral mucosa, and a smooth, red, painful dorsal tongue mucosa with papillary atrophy. These signs and symptoms actually rule out a diagnosis of BMS [16].

Another type of anaemia which can mimic BMS is pernicious anemia. This is a megaloblastic anemia caused by a lack of vitamin B12, which is due to a deficiency of the intrinsic factor responsible for 
the resorption of vitamin B12. In this disease, oral symptoms may include a burning or itching sensation of the oral mucosa, taste disturbances, intolerance to wearing prostheses, and occasionally dry mouth. The clinical examination can reveal atrophy of the papillae of the dorsum tongue. In advanced cases, the tongue may have a smooth, bright red surface, as reported in a single case report [17].

In BMS cases with deficiencies of zinc levels, the replacement therapy improved the symptoms but did not remit it completely [18].

\section{Vitamin deficiencies other than B12}

In a study among 70 BMS cases, without clinically mucosal changes, levels of a number of serum vitamins has been determined. In 28 cases (40\%) a reduction of vitamins B1, B2, B6 or a combination of these vitamins was found and no case was anaemic or iron deficient. None of the 70 BMS cases showed a scarcity in vitamins A, C, D or E. In a control group rhyming subjects without complaints of oral burning, vitamins reduced level was detected in only $7 \%$. The vitamin-deficient BMS group of 28 cases was given proper remedy. The non-vitamin-deficient BMS cases were given identical vitamin regimens. Of the 28 vitamin-deficient cases, a remarkable 24 cases were asymptomatic after 1 month and remained so after 3 months. No remission was reported in any of the non-vitamin-deficient cases after 1 month or after 3 months [16].

\section{Diabetes mellitus}

Diabetes has been connected to BMS based on the underlying mechanism of peripheral neuropathy (as burning feet) which it induces in time. Some authors consider that this comorbidity in untreated or poorly controlled can mimic the BMS and it was noticed that a better control of the main disease does improve the pain [7]. Another possible connection with diabetes is the experience of salivary dysfunction, which can lead to a decrease in salivary flow and change in saliva composition. Many studies have detected impaired salivary function in adults with diabetes, the universal prevalence of xerostomia in diabetic patients varies between $34 \%$ and 51\% [19]. Xerostomia can lead to many problems, such as difficulty eating, swallowing and speaking and can favour oral candidiasis. It may have a negative effect on patients' quality of life. The etiology is a mystery, but may be related to polyuria, neuropathy and microvascular changes and also changes in the salivary glands.

There is also a significant relationship between xerostomia and glucose levels in saliva, and the highest level of salivary dysfunction is observed in cases with poor glycemic control [19]. There is a correlation between immune factors related to BMS and increased sedimentation rate and increased salivary IgA encountered in diabetes patients [20].

\section{Endocrine disorders}

Gonadal hormones

Despite the unspecified etiology of this discomfort, the experiments confirmed the influence of the underlying hormonal factors [21].

Inconstant psychoendocrinological interactions may affect the oral mucosa and the severity of oral burning sensation in patients with postmenopausal BMS [22]. This is explained by the changes in the sensory pathway caused by decrease of estrogen levels [7]. Although the response of the vaginal epithelium to estrogen, examined by exfoliative cytology and using an immunohistochemical test, is quite distinct and the response of the oral epithelium is minimal [22].

It has been appreciated that BMS is triggered by a nervous system damage induced by neurotoxic factors on small fibers and basal ganglia in an environment of decreased neuroprotective gonadal hormones and increased levels of stress hormones, typical of menopause. It mainly affects women, especially after menopause, when its prevalence can be between $18 \%$ and $33 \%$ [23]. In postmenopausal women, BMS typically occurs without significant oral lesions, normal laboratory findings, and in association with psychological factors such as depression, which may be due to a range of factors, such as hormonal factors. In addition, neuropathic changes, oral phantom pain, and nerve inflammation may be predisposing factors for BMS [24].

\section{Hashimoto 's thyroiditis}

Hashimoto's thyroiditis is an immune thyroid disease that accounts for 30\% of aggressive thyroid disease. It is more common in women (about 5-10\%) and in patients with other types of self-aggressive diseases [25]. Both genetics and environmental factors contribute to the etiological causes of this disease.

In untreated patients with Hashimoto's thyroiditis, the levels of TSH in hypothyroidism, Anti-TPO, Anti-TG, Free T3, Free T4 modified indices were associated with BMS-like symptoms. Therefore, it seems the levels of various hormones, especially in hypothyroidism, is among the factors affecting BMS. Besides, the increased levels of Anti-TPO, Free T3, and TSH indices was associated with the BMS intensity [26].

\section{Hypothalamic-pituitary-adrenal axis}

Circadian rhythm dysfunction has been speculated to underlie anxiety, depression, sleep disturbances, hypothalamic-pituitary-adrenal axis dysfunction, and chronic pain experienced by patients 
with BMS [27]. Disruptions in the circadian rhythm affect pain perception, mood and it may affect hypothalamic-pituitary-adrenal axis [28].

\section{Side effects of medications}

In the literature there are case reports of BMS considered to be caused by drug intake. The angiotensin-converting enzyme inhibitors are the most commonly reported but the pathogenic mechanism is not yet fully determined [29,30,31]. Some authors incriminate the inflammatory reaction induced by bradykinin increased level. Other drugs reported to favor BMS-like symptoms are antipsychotics, antiretrovirals, and benzodiazepines [29]. This association between BMS and other drugs is controversial as it may be connected with the dose, the pharmacology, other psychogenic factors and the changes related to age [29].

\section{Psychiatric disorders}

Psychogenic factors frequently associated with BMS symptoms are anxiety, depression, or personality disorders. This pathogenic mechanism may be related to dopaminergic hypofunction. Analyzing the history of psychiatric disorders, some authors noticed that the onset of BMS was preceded by major depression, generalized anxiety disorder, and painful conditions other than orofacial disorders in almost $80 \%$ of cases [32]. When compared to controls, in BMS patients the sleep quality is reduced [33].

\section{BURNING MOUTH LIKE-SYNDROME AND ORAL MUCOSA RELATED CONDITIONS}

Although the symptoms of BMS are diagnosed by a complete and minutious anamnesis, a thorough examination of the oral cavity and, in particular, of the oral mucosa, is mandatory in the diagnostic procedure. Oral examination requires an adequate light source. Partial or complete dentures must be removed. For a proper examination of the tongue, the patient will be asked to extend the tongue and to move it through the oral cavity in order to permit the visual examination of all the mucosa [34]. Some oral benign conditions can be observed in BMS patients.

\section{Oral candidiasis}

Oral candidiasis can be developed by many predisposing factors, including xerostomia. Salivary dysfunction in these patients may contribute to the increased transport of fungi. Candida-related lesions include pseudomembranous and atrophic mucosal areas, angular cheilitis and median rhomboid glossitis. In at least a quarter of the population C. albicans is present in the normal oral flora without causing visible mucosal clinical changes. Can- dida growth can be enhanced by several local and general factors, resulting in penetration into the upper layers of the epithelium. Candidiasis is more common in smokers, in diabetic patients with poor glycemic control, in dentures wearers, in patients who use local and general steroids and antibiotics $[35,36,37]$. The diagnosis of oral candidiasis is established by clinical criteria and mycological confirmation. The treatment is mainly topical with antifungal agents.

\section{Atrophy of the oral mucosa}

A study of 20 BMS patients and 20 healthy control subjects analyzed oral smears collected from the normal clinical appearance mucosa in order to detect cytological and cytomorphometric techniques. The authors concluded that these changes are likely to be associated with epithelial atrophy and unregulated maturation process of the oral mucosa that may contribute to oral symptoms [38].

The fungiform papillae of the anterior dorsal tongue can become inflamed ('lingual papillitis'), producing a reddish aspect of the tongue surface and often accompanied by burning sensations which resemble BMS symptoms but disappear in a short period [39].

\section{Anatomical variants of the tongue}

Self-observation of oral mucosal conditions may increase the patient's attention on this area and aggravate pre existing symptoms with or without objective causes.

Geographical tongue, also called erythema migrans, is a benign condition of unknown etiology, characterized by recurrent atrophic areas on the dorsum and lateral edges of the tongue [40]. Fissured tongue is another normal variant of the dorsal tongue. Both clinical entities require no treatment and are unanimously accepted as benign conditions which carry no risk.

Median rhomboid glossitis is a condition of the dorsal tongue mucosa located on the midline, anterior to the foramen caecum. The areas are red flat or slightly prominent or nodular, rhomboid or oval shape [34].

Lingual tonsil is a benign hyperplastic lymphoid tissue ("lymphoid hyperplasia") located in the posterior margin of the tongue. It is most frequently bilaterally and increases in volume if dental foci are present or in case of mechanical dental injury of the areas.

\section{ODONTOGENIC CAUSES}

In BMS patients any dental treatment that is not really indicated should be avoided until a certain diagnosis. Some of the local causes of BMS can be allergy to dental materials, galvanism, dental treat- 
ment, etc. Oral galvanism refers to an electrochemical reaction between different dental metal restorations in the presence of a conductive solution, such as saliva. The size of the currents can be calculated after measuring the potentials and polarizations of the metal restorations.

Prosthesis or dentures problems related to occlusion, stability and temporomandibular joint are considered to be a cause observed in $50 \%$ of BMS patients. In almost all patients, complete resolution of symptoms was obtained after removal of the prosthesis defects [41].

\section{CONCLUSIONS}

BMS is a complex disease that affects not only one organ or structure, but the body as a whole. The clinician should be prepared for establishing differential diagnosis and know the difference between primary BMS and BMS-like symptoms.

There are symptoms that mimic BMS in systemic diseases such as diabetes, Hashimoto 's thyroiditis and also circadian rhythm dysfunction, in malfunction of hypothalamic-pituitary-adrenal axis, allergies, etc.

In addition, the local factors should be taken into consideration and the oral mucosa be evaluated for unusual features, candidiasis or recent dental treatment, galvanism.

Most affected cases are over 50 years old with a frequency higher in women than in men. Because the etiology of BMS is multifactorial, treatment can only be distinctive, and is aimed at relieving symptoms. The complexity of BMS symptoms and associated psychosocial infirmities, anxiety and depression raise the need for a multidisciplinary and individualised approach.

\section{Acknowledgement}

This work was supported by a grant of the Romanian Ministry of Education and Research, CCCDI UEFISCDI, project number PN-III-P2-2.1-PED-20191339 within PNCDI III.(Contract number 564PED/ 2021).

\section{REFERENCES}

1. World Health Organization. International classification of diseases, 11th revision. 2018. Available at: https://icd.who.int/browse11/I-m/ en\#/http://id.who.int/icd/entity/618998878.

2. Lamb AB, Lamey PJ, Reeve PE. Burning mouth syndrome: psychological aspects. Br Dent J. 1988;165(7):256-60.

3. Lamey P. Burning mouth syndrome. Dermatol Clin. 1996; 14(2):339-54.

4. Lamey PJ, Lamb AB, Hughes A, Milligan KA, Forsyth A. Type 3 burning mouth syndrome: psychological and allergic aspects. J Oral Pathol Med. 1994;23:216-9.

5. Scala A, Checchi L, Montevecchi M, Marini I, Giamberardino MA. Update on burning mouth syndrome: overview and patient management. Crit Rev Oral Biol Med. 2003;14(4):275-91.

6. Jääskeläinen SK. Is burning mouth syndrome a neuropathic pain condition? Pain. 2018;159(3):610-3.

7. Renton T. Burning mouth syndrome. Rev Pain. 2011;5(4):12-7.

8. Romeo U, Del Vecchio A, Capocci M, Maggiore C, Ripari M. The low level laser therapy in the management of neurological burning mouth syndrome. A pilot study. Ann Stomatol (Roma). 2010;1(1):14-8.

9. Headache Classification Committee of the International Headache Society (IHS). The International Classification of Headache Disorders, 3rd edition. Available at: https://ichd-3.org/13-painful-cranialneuropathies-and-other-facial-pains/13-11-persistent-idiopathicfacial-pain-pifp/.

10. Scala A, Checchi L, Montevecchi M, Marini I, Giamberardino MA. Update on burning mouth syndrome: overview and patient management. Crit Rev Oral Biol Med. 2003;14(4):275-91.

11. 11.Chiang $C P$, Wu YC, Wu YH, Chang JY, Wang YP, Sun A. Gastric parietal cell and thyroid autoantibodies in patients with burning mouth syndrome. J Formos Med Assoc. 2020 Dec;119(12):1758-1763.

12. Lechien JR, Hans S, De Marrez LG, Dequanter D, Rodriguez A, Muls V, Ben Abdelouahed F, Evrard L, Maniaci A, Saussez S, Bobin F. Prevalence and Features of Laryngopharyngeal Reflux in Patients with Primary Burning Mouth Syndrome. Laryngoscope. 2021; 131(10):E2627-E2633.

13. Sherman A III, Zamulko A. Burning tongue as initial presentation of celiac disease in an elderly woman: a case report. SD Med. 2016;69(6):253-5.

14. Powell A, Nelson KN, Awosika O, Rengifo-Pardo M, Ehrlich A. Burning mouth syndrome and contact dermatitis. Dermatitis. 2020; 31(4):238-43.

15. Halac G, Tekturk P, Eroglu S, Cikrikcioglu MA, Cimendur O, Kilic E, et al. Burning mouth syndrome: evaluation of clinical and laboratory findings. Ideggyogy Sz. 2016;69(7-8):269-74.

16. Lehman JS, Bruce AJ, Rogers RS. Atrophic glossitis from vitamin B12 deficiency: a case misdiagnosed as burning mouth disorder. J Periodontol. 2006;77(12):2090-2.

17. Sardella A, Lodi G, Demarosi F, Uglietti D, Carrassi A. Causative or precipitating aspects of burning mouth syndrome: a case-control study. J Oral Pathol Med. 2006;35(8):466-71.

18. Cho GS, Han MW, Lee B, Roh JL, Choi SH, Cho KJ, Nam SY, Kim SY. Zinc deficiency may be a cause of burning mouth syndrome as zinc replacement therapy has therapeutic effects. J Oral Pathol Med. 2010 Oct;39(9):722-7.

19. Rohani B. Oral manifestations in patients with diabetes mellitus. World J Diabetes. 2019 Sep 15;10(9):485-489.

20. Bookout GP, Ladd M, Short RE. Burning Mouth Syndrome. 2021 Feb 17. In: StatPearls [Internet]. Treasure Island (FL): StatPearls Publishing; 2021 Jan.

21. Crow HC, Gonzalez Y. Burning mouth syndrome. Oral Maxillofac Surg Clin North Am. 2013 Feb;25(1):67-76.

22. Kang JH, Kim YY, Chang JY, Kho HS. Relationships between oral MUC1 expression and salivary hormones in burning mouth syndrome. Arch Oral Biol. 2017 Jun;78:58-64.

23. Zakrzewska J, Buchanan JA. Burning mouth syndrome. BMJ Clin Evid. 2016 Jan 7;2016:1301.

24. Minguez-Sanz MP, Salort-Llorca C, Silvestre-Donat FJ, Etiology of burning mouth syndrome: a review and update. Med Oral Patol Oral Cir Bucal. 2011 Mar 1;16(2):e144-8. 
25. Pyzik A, Grywalska E, Matyjaszek-Matuszek B, Roliński J. Immune disorders in Hashimoto's thyroiditis: what do we know so far? J Immunol Res. 2015;2015:979167.

26. Talattof Z, Dabbaghmanesh MH, Parvizi Y, Esnaashari N, Azad A. The Association between Burning Mouth Syndrome and Level of Thyroid Hormones in Hashimoto's Thyroiditis in Public Hospitals in Shiraz, 2016. J Dent (Shiraz). 2019 Mar;20(1):42-47.

27. Panda S. The circadian code: lose weight, supercharge your energy, and transform your health from morning to midnight. Rodale Books, 2020.

28. Ritchie A, Kramer JM. Recent Advances in the Etiology and Treatment of Burning Mouth Syndrome. J Dent Res. 2018 Oct;97(11):1193-1199.

29. Azzi L, Veronesi G, Tagliabue A, et al. Is there an association between drugs and burning mouth syndrome? A case-control study. Oral Dis. 2019;25:1634-1644.

30. Raghavan SA, Puttaswamiah RN, Birur PN, Ramaswamy B, Sunny SP. Antidepressant-induced Burning Mouth Syndrome: A Unique Case. Korean J Pain. 2014 Jul;27(3):294-6.

31. Obara T, Naito H, Nojima T, Koga H, Nakao A. Burning Mouth Syndrome Induced by Angiotensin-Converting Enzyme Inhibitors. Cureus. 2020;12(11):e11376.

32. Taiminen T, Kuusalo L, Lehtinen L, Forssell H, Hagelberg N, et al. Psychiatric (axis I) and personality (axis II) disorders in patients with burning mouth syndrome or atypical facial pain. Scand J Pain. 2011 Oct 1;2(4):155-160.
33. Adamo D, Sardella A, Varoni E, Lajolo C, et al. The association between burning mouth syndrome and sleep disturbance: A case-control multicentre study. Oral Dis. 2018 May;24(4):638-649.

34. Tovaru S, Tovaru M, Costache M, Demarossi F. Medicină şi patologie orală, vol II. Q Med Publishing, 2014.

35. Al-Maskari AY, Al-Maskari MY, Al-Sudairy S. Oral Manifestations and Complications of Diabetes Mellitus: A review. Sultan Qaboos Univ Med J. 2011;11:179-186.

36. Ship JA. Diabetes and oral health: an overview. J Am Dent Assoc 2003;134 Spec No:4S-10S.

37. Khan T. Oral manifestations and complications of diabetes mellitus: A review. Int J Med Health Res. 2018;4:50-52.

38. Wandeur T, de Moura SA, de Medeiros AM, Machado MA, Alanis LR, Grégio AM, Trevilatto PC, de Lima AA. Exfoliative cytology of the oral mucosa in burning mouth syndrome: a cytomorphological and cytomorphometric analysis. Gerodontology. 2011;28(1):44-8.

39. Kalogirou EM, Tosios KI, Nikitakis NG, Kamperos G, Sklavounou A. Transient lingual papillitis: a retrospective study of 11 cases and review of the literature. J Clin Exp Dent. 2017;9(1):e157-62.

40. Su N, Poon R, Liu C, Dewan C, Darling M, Grushka M. Taste and pain response in burning mouth syndrome with and without geographic tongue. J Oral Facial Pain Headache. 2020;34(3):217-21.

41. Main DM, Basker RM. Patients complaining of a burning mouth. Further experience in clinical assessment and management. Br Dent J. 1983;154(7):206-11. 